\title{
Lumikot : Fast Auroral Transients During the Growth Phase of Substorms
}

McKay, D.

2019-07-16

McKay , D , Paavilainen , T , Gustavsson, B , Kvammen , A \& Partamies , N 2019 , '

Lumikot : Fast Auroral Transients During the Growth Phase of Substorms ', Geophysical

Research Letters, vol. 46 , no. 13 , pp. 7214-7221 . https://doi.org/10.1029/2019GL082985

http://hdl.handle.net/10138/311455

https://doi.org/10.1029/2019GL082985

publishedVersion

Downloaded from Helda, University of Helsinki institutional repository.

This is an electronic reprint of the original article.

This reprint may differ from the original in pagination and typographic detail.

Please cite the original version. 


\section{Geophysical Research Letters}

\author{
RESEARCH LETTER \\ 10.1029/2019GL082985 \\ Key Points: \\ - Small, transient auroral features \\ (Lumikot) have been observed in the \\ growth phase of substorms \\ - They move bidirectionally along the \\ boundary between the bright arc and \\ the diffuse aurora \\ - They map magnetically to the \\ boundary region between the \\ plasmasheet and outer radiation belt
}

Supporting Information:

- Supporting Information S1

Correspondence to:

D. McKay,

derek.mckay@norut.no

Citation:

McKay, D., Paavilainen, T.,

Gustavsson, B., Kvammen, A., \&

Partamies, N. (2019). Lumikot: Fast auroral transients during the growth phase of substorms. Geophysical Research Letters, 46, 7214-7221. https://doi.org/10.1029/2019GL082985

Received 23 MAR 2019 Accepted 20 JUN 2019 Accepted article online 25 JUN 2019 Published online 9 JUL 2019

(C)2019. American Geophysical Union. All Rights Reserved.

\section{Lumikot: Fast Auroral Transients During the Growth Phase of Substorms}

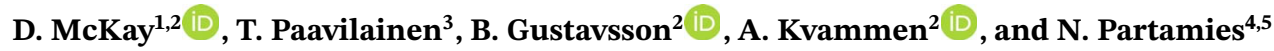 \\ ${ }^{1}$ NORCE Norwegian Research Centre AS, Tromsø, Norway, ${ }^{2}$ Department of Physics and Technology,UiT-The Arctic \\ University of Norway, Tromsø, Norway, ${ }^{3}$ Teaching and Learning Services,University of Helsinki, Helsinki, Finland, \\ ${ }^{4}$ Department of Arctic Geophysics, The University Centre in Svalbard, Longyearbyen, Norway, ${ }^{5}$ Birkeland Centre for \\ Space Science, Bergen, Norway
}

Abstract The development of a magnetospheric substorm may be classified into three phases: growth, expansion, and recovery. The growth phase is important as it includes processes that lead to the expansion. In a recent growth-phase study, a type of fast discrete auroral transient phenomena-referred to as Lumikot-were observed. The Lumikot are several kilometers across and move in the high-energy precipitation region, parallel to the main growth-phase arc, with both east-west and west-east directions of travel during the same event. Their apparent transverse movement and quasi-stable intensity make them distinct from cooccurring optical pulsating aurorae. Comparison to other studies show that they occur in the cosmic noise absorption region and it is likely that the Lumikot are colocated with high-energy particle populations on the boundary between the outer radiation belt and the plasmasheet.

\section{Introduction}

An auroral substorm takes place in a se quence of three phases: the growth, expansion, and recovery phase. The initial stage of the substorm is the growth phase, lasting 1-2 hr (McPherron, 1970). During this time, a narrow, east-west aligned growth-phase arc drifts equatorward and brightens prior to the poleward expansion at the substorm onset. The expansion phase is accompanied by an increase in high-energy particle precipitation (Wing et al., 2013), which originates from the plasma sheet (Wing et al., 2013). Lower-energy electrons $(<10 \mathrm{keV})$ cause the optical emission, while higher-energy electrons penetrate deeper in the atmosphere and can be detected via cosmic (radio) noise absorption (CNA).

The main growth-phase arc is discrete aurora. However, a broad region of diffuse emission often exists on the equatorward side of this arc, shown to be proton aurora (Ono et al., 1987). The relationship between the discrete and diffuse emission was studied by McKay et al. (2018). They found an arc of CNA equatorward of the optical emission and contained within diffuse aurora. The offset between the growth phase and the CNA arc is nearly uniform during the equatorward progression of the growth-phase arc. McKay et al. (2018) also observed optical pulsating aurora between the diffuse emission and the main growth-phase arc, colocated with CNA. In the same study, fast-moving auroral patches were seen to traverse the sky, in the same region where the optical pulsations later appeared. These patches are referred to as Lumikot (singular: Lumikko, from the Finnish word for weasel).

This paper characterizes the Lumikot precipitation and their appearance. In particular, the particle precipitation source region is inferred by estimating the altitude and position of the Lumikot with respect to the growth phase and CNA arc. Furthermore, the plasmasheet projection of the Lumikot is approximated by employing the Tsyganenko T96 magnetospheric model. The association between the Lumikot and the optical aurora pulsations is discussed as well.

\section{Instrumentation}

For this study, two instruments of the Finnish Meteorological Institute's All-Sky Camera (FMI-ASC) network were used, located at Kilpisjärvi $\left(20.78^{\circ} \mathrm{E}, 69.05^{\circ} \mathrm{N}\right.$, elevation $\left.480 \mathrm{~m}\right)$ and Abisko $\left(18.82^{\circ} \mathrm{E}, 68.36^{\circ} \mathrm{N}\right.$, elevation $370 \mathrm{~m}$ ). The FMI-ASCs (Sangalli et al., 2011) use electron multiplication CCDs and filter wheels for the main auroral emission lines at 427.8, 557.7, and $630.0 \mathrm{~nm}$. A standard imaging mode of 20 images per minute also includes images using background filters, dark frames, and panchromatic images. The cadence of any single wavelength is irregular. Exposure times for 427.8- and 557.7-nm images are 1.2 and $0.8 \mathrm{~s}$, 


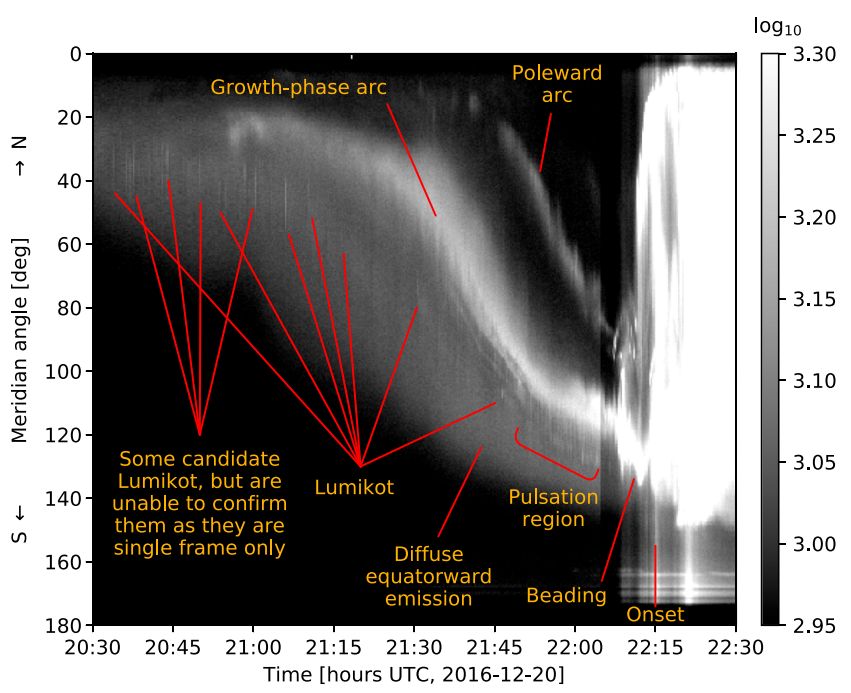

Figure 1. Keogram from the Kilpisjärvi all-sky camera showing the features of the substorm and the location of the Lumikot and Lumikot candidates therein. respectively. Displayed grayscale levels for all images are in arbitrary analog-to-digital units (ADU) but are linear to the received luminance. The cameras use fish-eye optics to generate all-sky images with $512 \times$ 512 pixels. At auroral altitudes of $110 \mathrm{~km}$, this results in a near-zenith resolution of less than $1 \mathrm{~km}$ per pixel. The alignment of the FMI-ASCs at Kilpisjärvi and Abisko, respectively, is $7.53^{\circ}$ and $6.50^{\circ}$ east of geographic north. Magnetic north is $\approx 9.5^{\circ}$ east of north for the observations in this paper. The camera lens' projection is an equidistant one (linear to pixels), and after geometric calibration the pointing error is less than $0.25^{\circ}$ for all pixels.

\section{Observations and Results}

Details about the geomagnetic conditions of the substorm from 20 December 2016 (commencing at 20 UT, and continuing to 21 December 201603 UT) can be found in McKay et al. (2018). This isolated event was preceded by a quiet-time period of $20 \mathrm{hr}$. The planetary K index was $K_{p}=1$ and the solar wind speed from 18:00-21:00 UT was slow ( $v=$ $367 \pm 5 \mathrm{~km} / \mathrm{s}$ ). The interplanetary magnetic field was steadily negative with $B_{z}=-3.2 \pm 0.6 \mathrm{nT}$ during the entire growth phase. Although these parameters describe the overall activity level, the magnetic field measurements (even the local ones) do not capture the disturbances related to the small-scale transient auroral signatures.

A broad diffuse arc formed over Kilpisjärvi at 19:22 UT with a bright growth-phase arc appearing at 20:58 UT on the northern edge of the diffuse emission. The arc system drifted equatorward at the speed of $71 \pm 7$ $\mathrm{m} / \mathrm{s}$. During the evolution of the growth phase, small patches of aurora (Lumikot) were seen just south of the growth-phase arc, rapidly translating parallel to the arc across the field of view. The classification criteria is that the patch must be $3 \sigma$, finite in extent, moving laterally, and seen in three consecutive frames (in order to confirm this movement). This region of the Lumikot corresponds to high-energy precipitation, as indicated by CNA measured by the Kilpisjärvi Atmospheric Imaging Receiver Array (KAIRA; Figures 2 and 3 in McKay et al., 2018).

Multiple Lumikot were observed between 19:25 UT (when the cloud cover first cleared) and 22:10 UT (onset) within the CNA region, shown in Figure 1.

Both east-to-west and west-to-east directions of travel were observed. Two examples, \#1 and \#2, are shown in Figures 2 and 3, respectively. In each figure a sample all-sky frame is shown at the top. A region around each Lumikko, marked with a red box, has been extracted from each individual frame. These are presented below in chronological order, with the time, filter wavelength, and exposure time included. Because of the imaging sequence, the time between slices is nonuniform.

In addition to the 20 December 2016 event, six other substorms used in the previous study were investigated and Lumikot were found in two of them: 8 February 2015 and 18 February 2017. A total of $20 \mathrm{E} \rightarrow$ W Lumikot and $17 \mathrm{~W} \rightarrow$ E Lumikot were detected, which represents a lower limit of the occurrence rate. Lumikot-like features were seen in many single frames, but it was not possible to discern if they travel too fast for the cadence time or if they appear and disappear without positional movement. These cases were discarded.

\subsection{Altitude Determination}

The simultaneous imaging and the overlapping fields of view of the two cameras permitted altitude determination of the Lumikot. A background subtraction was needed to isolate the Lumikko emission. To estimate the background for each Lumikko, a four-step procedure was used: (1) filtering the image using a $3 \times 3$ median filter; (2) manually defining an area that encloses the Lumikko; (3) cutting out the enclosed area; (4) performing a 2-D interpolation of the empty area. The resulting image is essentially identical to the original image but with the Lumikko emission removed. The Lumikko was thereafter isolated by subtracting the interpolated background image from the original image. This procedure is only effective if the Lumikko is positioned on a smooth background, such as the diffuse aurora is in this case (Figures 2 and 3). 

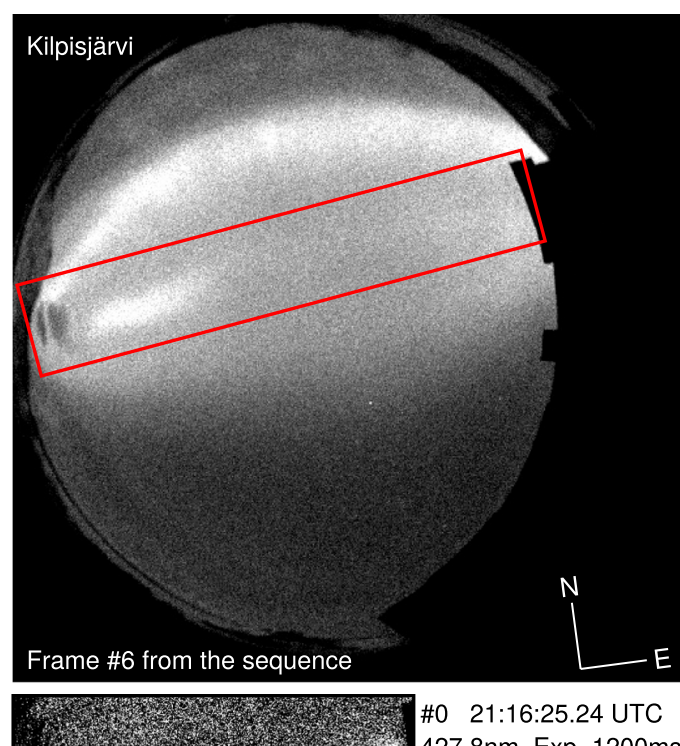

$427.8 \mathrm{~nm}$, Exp $=1200 \mathrm{~ms}$

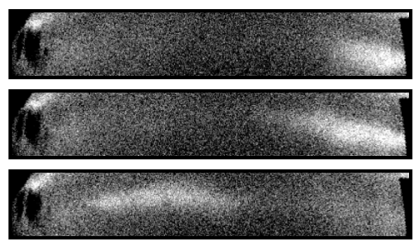

\#1 21:16:27.64 UTC

$557.7 \mathrm{~nm}, \operatorname{Exp}=800 \mathrm{~ms}$

\#2 21:16:30.24 UTC

$557.7 \mathrm{~nm}, \mathrm{Exp}=800 \mathrm{~ms}$

\#3 21:16:40.81 UTC

$557.7 \mathrm{~nm}, \operatorname{Exp}=800 \mathrm{~ms}$

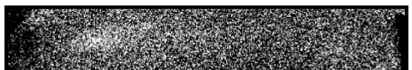

\#4 21:16:42.72 UTC

$427.8 \mathrm{~nm}, \mathrm{Exp}=1200 \mathrm{~ms}$

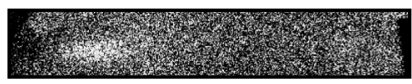

\#5 21:16:45.24 UTC

$427.8 \mathrm{~nm}, \mathrm{Exp}=1200 \mathrm{~ms}$

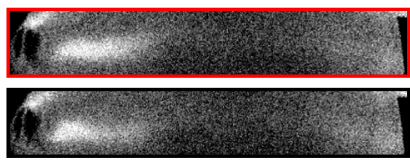

\#6 21:16:47.64 UTC

$557.7 \mathrm{~nm}, \mathrm{Exp}=800 \mathrm{~ms}$

\#7 21:16:50.25 UTC

$557.7 \mathrm{~nm}, \operatorname{Exp}=800 \mathrm{~ms}$

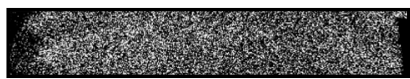

\#8 21:16:55.81 UTC

$427.8 \mathrm{~nm}, \mathrm{Exp}=1200 \mathrm{~ms}$
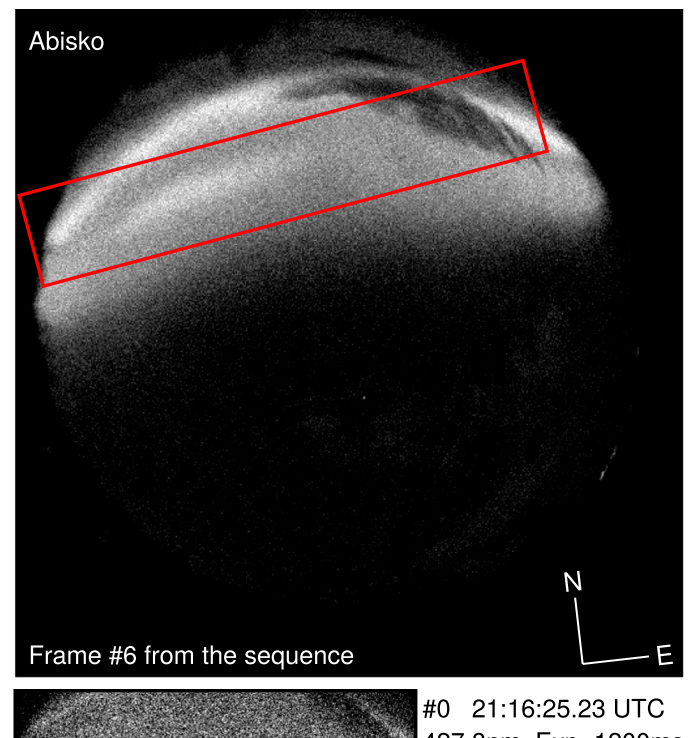

$427.8 \mathrm{~nm}, \mathrm{Exp}=1200 \mathrm{~ms}$

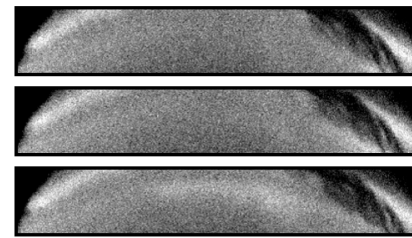

\#1 21:16:27.59 UTC

$557.7 \mathrm{~nm}$, Exp $=800 \mathrm{~ms}$

\#2 21:16:30.23 UTC

$557.7 \mathrm{~nm}, \operatorname{Exp}=800 \mathrm{~ms}$

\#3 21:16:40.80 UTC

$557.7 \mathrm{~nm}, \operatorname{Exp}=800 \mathrm{~ms}$

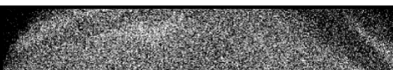

\#4 21:16:42.66 UTC

$427.8 \mathrm{~nm}, \mathrm{Exp}=1200 \mathrm{~ms}$

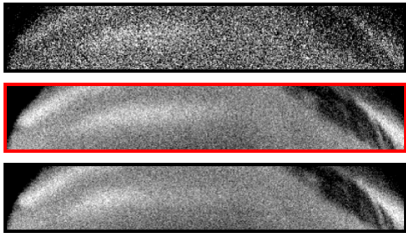

\#5 21:16:45.23 UTC

$427.8 \mathrm{~nm}, \mathrm{Exp}=1200 \mathrm{~ms}$

\#6 21:16:47.59 UTC

$557.7 \mathrm{~nm}, \operatorname{Exp}=800 \mathrm{~ms}$

\#7 21:16:50.23 UTC

$557.7 \mathrm{~nm}, \mathrm{Exp}=800 \mathrm{~ms}$

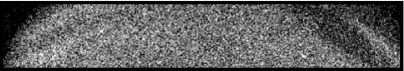

\#8 21:16:55.80 UTC $427.8 \mathrm{~nm}$, Exp $=1200 \mathrm{~ms}$

Figure 2. Example Lumikko \#1, for the (left) Kilpisjärvi and (right) Abisko all-sky cameras, with apparent east-to-west motion. The top panel in each column is an all-sky image. Individual, time-ordered slices at green and blue wavelengths are shown below.

The coordinates of each Lumikko were determined by searching for an altitude, $z$, for which the Lumikko projections from Abisko and Kilpisjärvi were overlapping and thus minimizing the least squares error function, $E(z)$.

$$
E(z)=\arg \min _{z}\left(\frac{1}{\alpha(z)} \sum_{i, j}\left[P_{A}(z,(i, j))-P_{K}(z,(i, j))\right]^{2}\right)
$$

where $P_{A}(z,(i, j))$ and $P_{K}(z,(i, j))$ are the normalized Lumikko projections to altitude $z$ from the background-subtracted images at Abisko and Kilpisjärvi; $(i, j)$ are the image pixel coordinates in longitude and latitude; $\alpha(z)$ is an altitude-dependent normalization factor, proportional to the total intensity of the projected Lumikko.

The projection of the images to the longitude-latitude shell at the altitude $z$ relies on precise geometric calibration of the camera fields of view. This is achieved by identifying stars in the image and fitting camera parameters (Gustavsson et al., 2008). Typically, detection of 200 stars results in $1 \sigma$ errors of approximately \pm 0.2 pixels.

The geographic coordinates of the Lumikot midpoints were similar in the Abisko and Kilpisjärvi projections when $E(z)$ was near the minimum. However, the shapes of the Lumikot projections were not identical, 

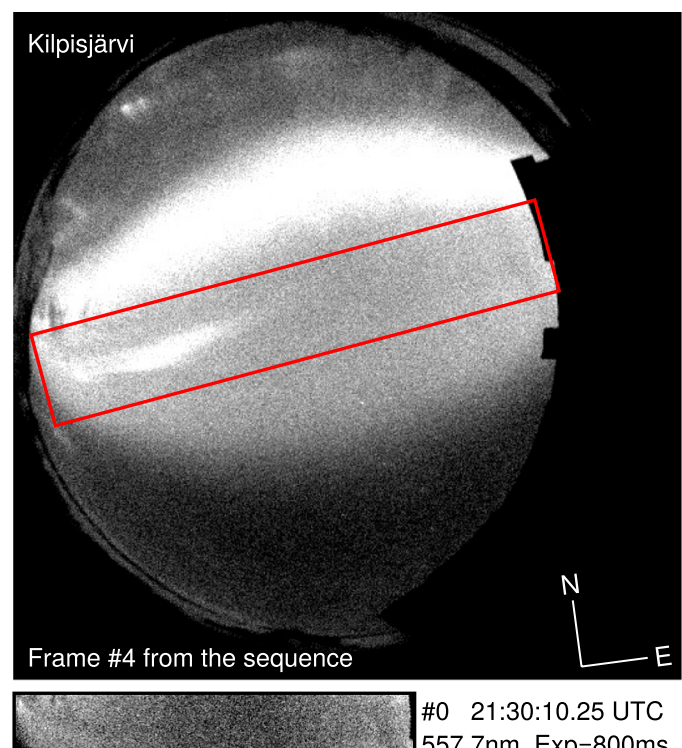

$557.7 \mathrm{~nm}, \operatorname{Exp}=800 \mathrm{~ms}$

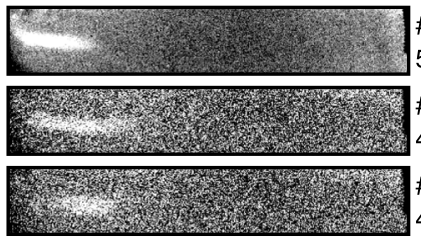

\#1 21:30:20.93 UTC

$557.7 \mathrm{~nm}, \operatorname{Exp}=800 \mathrm{~ms}$

\#2 21:30:22.83 UTC

$427.8 \mathrm{~nm}, \mathrm{Exp}=1200 \mathrm{~ms}$

\#3 21:30:25.25 UTC

$427.8 \mathrm{~nm}, \mathrm{Exp}=1200 \mathrm{~ms}$

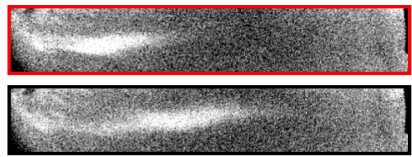

21:30:27.61 UTC

$557.7 \mathrm{~nm}, \mathrm{Exp}=800 \mathrm{~ms}$

\#5 21:30:30.25 UTC

$557.7 \mathrm{~nm}$, Exp $=800 \mathrm{~ms}$

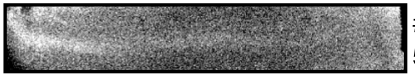

\#6 21:30:40.81 UTC

$557.7 \mathrm{~nm}$, Exp $=800 \mathrm{~ms}$

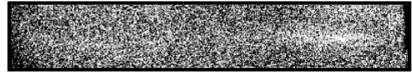

\#7 21:30:42.71 UTC

$427.8 \mathrm{~nm}, \mathrm{Exp}=1200 \mathrm{~ms}$

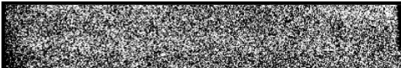

\#8 21:30:45.25 UTC

$427.8 \mathrm{~nm}, \mathrm{Exp}=1200 \mathrm{~ms}$
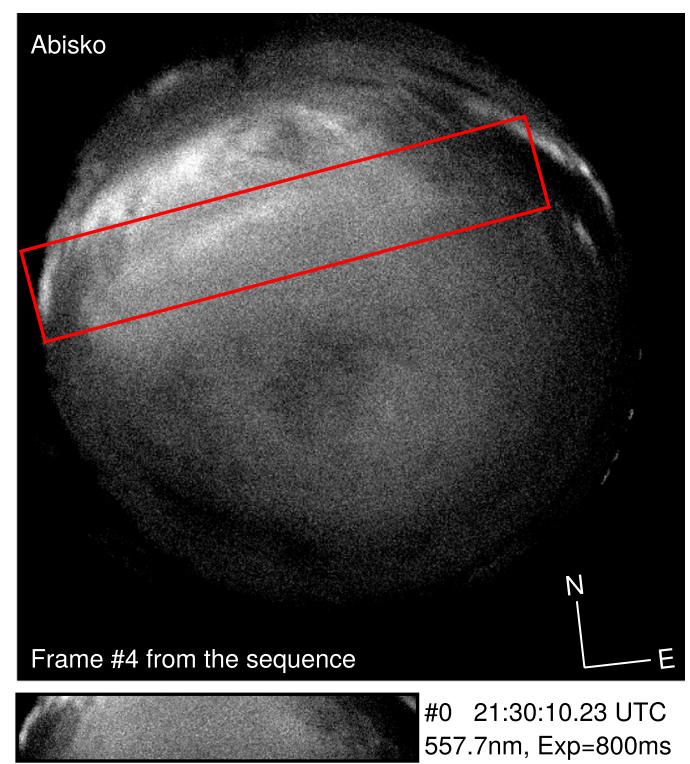

\#0 21:30:10.23 UTC $557.7 \mathrm{~nm}$, Exp $=800 \mathrm{~ms}$

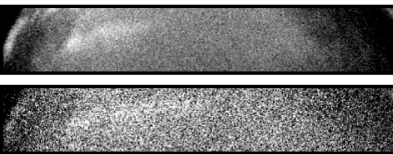

\#1 21:30:20.92 UTC $557.7 \mathrm{~nm}$, Exp $=800 \mathrm{~ms}$

\#2 21:30:22.78 UTC $427.8 \mathrm{~nm}, \operatorname{Exp}=1200 \mathrm{~ms}$

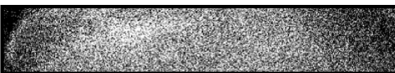

\#3 21:30:25.23 UTC $427.8 \mathrm{~nm}, \mathrm{Exp}=1200 \mathrm{~ms}$

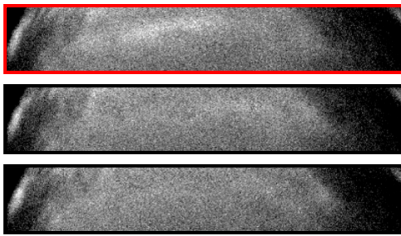

\#4 21:30:27.59 UTC $557.7 \mathrm{~nm}, \mathrm{Exp}=800 \mathrm{~ms}$

\#5 21:30:30.23 UTC $557.7 \mathrm{~nm}$, Exp $=800 \mathrm{~ms}$ \#6 21:30:40.80 UTC $557.7 \mathrm{~nm}$, Exp $=800 \mathrm{~ms}$

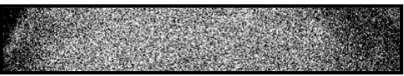

\#7 21:30:42.66 UTC $427.8 \mathrm{~nm}, \mathrm{Exp}=1200 \mathrm{~ms}$

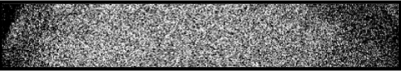

21:30:45.23 UTC $427.8 \mathrm{~nm}, \operatorname{Exp}=1200 \mathrm{~ms}$

Figure 3. Example Lumikko \#2, with apparent west-to-east motion.

as each Lumikko is not a horizontal emission layer, but a 3-D volume. The layer approximation results in a latitude difference between the projections. Nevertheless, the shell projection method is preferred as it utilizes information from the entire Lumikko, rather than one triangulation point. The disadvantage of the method is that the error function $(E(z))$ does not provide direct estimates of the coordinate deviation around the best-fit altitude. The projections corresponding to the altitudes with errors above $20 \%$ of the best-fit value show clear discrepancy, both below and above the best-fit altitude. A conservative confidence interval of altitudes within $20 \%$ of the best-fit error value was therefore used. The Lumikot image pairs in

Table 1

The Best-Fit Coordinate Values for the Sample Lumikot With Error Ranges

\begin{tabular}{lccc}
\hline Event & $\# 1 \mathrm{a}$ & $\# 1 \mathrm{~b}$ & $\# 2$ \\
time (UTC) & $21: 16: 48$ & $21: 16: 51$ & $21: 30: 28$ \\
\hline$x[\mathrm{~km}]$ & $-170[-166,-176]$ & $-201[-194,-207]$ & $-132[-133,-137]$ \\
$y[\mathrm{~km}]$ & $31[28,35]$ & $30[27,33]$ & $-9[-11,-8]$ \\
$z[\mathrm{~km}]$ & $118[114,124]$ & $120[115,125]$ & $115[110,121]$ \\
\hline
\end{tabular}

Note. The $x y$ coordinate ranges correspond to the $z$ coordinate ranges and are therefore not necessarily ordered from the lowest to highest value. 

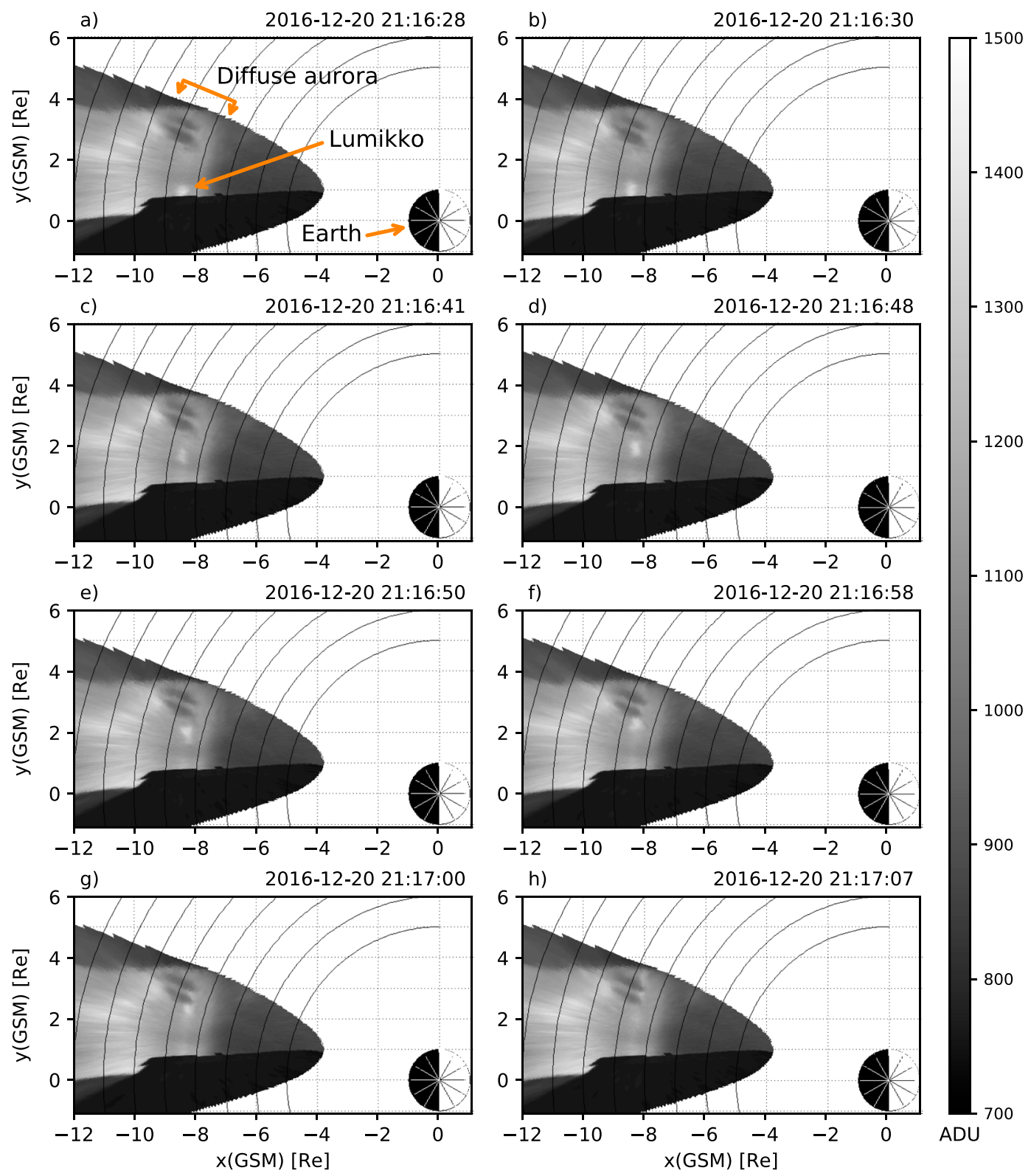

Figure 4. (a-h) The Kilpisjärvi images (projected to the plasmasheet) place the Lumikot at $x \approx-8 R_{e}, y \approx 2.2 R_{e}$. The growth-phase arc maps to the distance of approximately $10 R_{e}$ from the Earth. The dark structure at $y<1 R_{e}$ is not an auroral feature but a structure at the Kilpisjärvi site.

Figure 2 at 21:16:48 UTC (\#1a) and at 21:16:51 UTC (\#1b) and in Figure 3 at 21:30:28 UTC were used in the altitude determination. The altitude and horizontal coordinate results are summarized in Table 1 . The Lumikot $x y$ coordinates in Table 1 are transferred from degrees in longitude and latitude to kilometers north and east of Kilpisjärvi so that the velocity of Lumikko \#1 can then be estimated in appropriate units. The Lumikko $x y$ midpoint is defined as the mean center of two fitted asymmetrical 2-D Gaussians, where one 2-D Gaussian is fitted to the Abisko projection and the other is fitted to the Kilpisjärvi projection.

Lumikot take approximately $30-45 \mathrm{~s}$ to cross the sky. The apparent velocity of example \#1 based on the coordinates of \#1a and \#1b becomes $\bar{v}_{(x, y, z)} \approx[-12,0,1] \mathrm{km} / \mathrm{s} \pm[3,2,2] \mathrm{km} / \mathrm{s}$; that is, westward, with no appreciable change in the latitudes or heights (Table 1).

\subsection{Projection of Lumikot Onto the Plasmasheet}

The example Lumikko from Figure 2 was projected to the location along the magnetic field lines, which is the farthest distance from Earth, which should be the estimated location of the cross-tail current sheet, or 

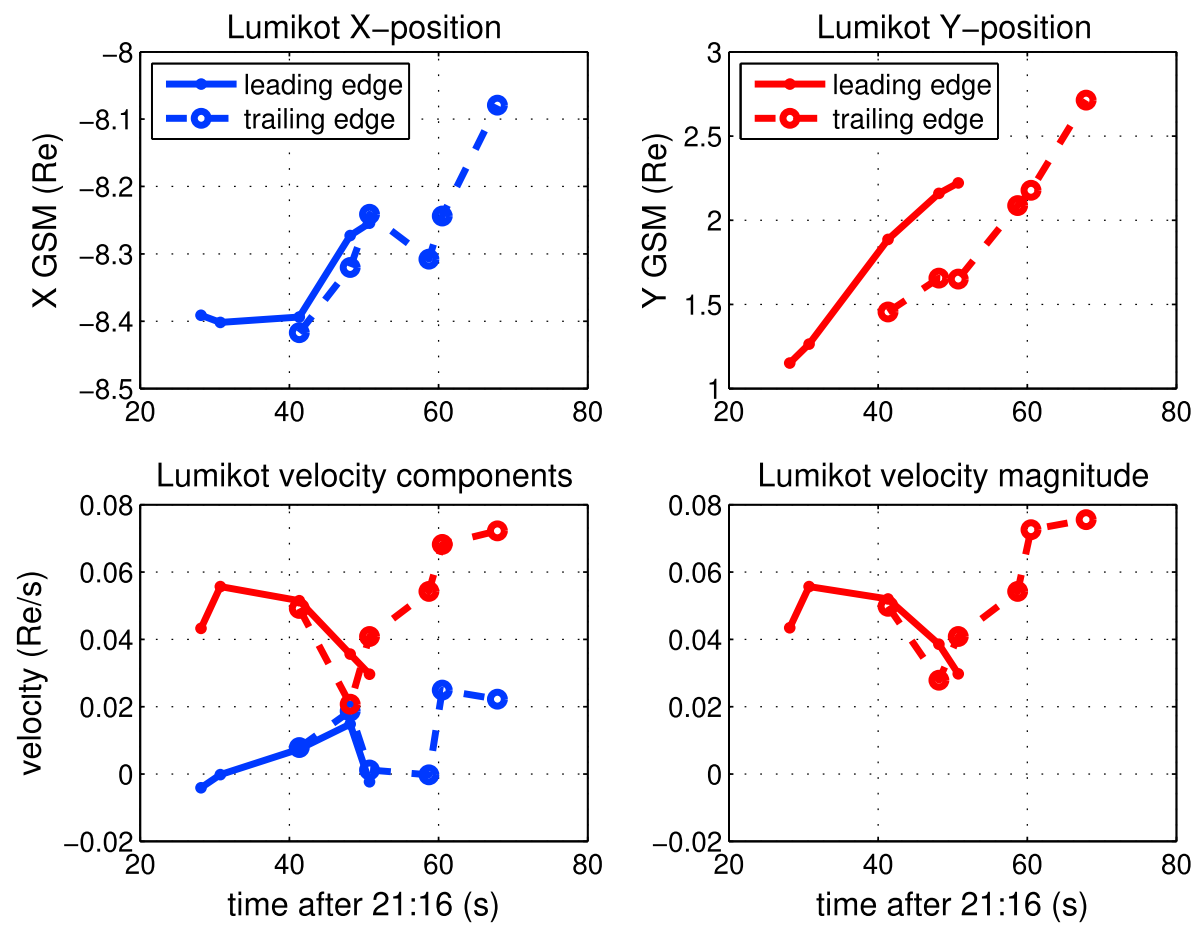

Figure 5. The magnetospheric $X$ and $Y$ coordinates of Lumikot at 21:16-21:17 UT are shown in the upper panels. The $X$ and $Y$ velocity components are plotted in the bottom left while the speed is shown in the bottom right panel.

plasmasheet, by first projecting the images to an initial altitude of $112 \mathrm{~km}$. Magnetic coordinates were calculated using aacgmv2 (Shepherd, 2014) Python libraries for the epoch 20 December 2016, giving Kilpisjärvi $=102.39^{\circ} \mathrm{E}, 66.06^{\circ} \mathrm{N}$ and Abisko $=100.35^{\circ} \mathrm{E}, 65.42^{\circ} \mathrm{N}$. The magnetic field lines were then traced from these locations into the magnetosphere using the Tsyganenko T96 magnetospheric model (Tsyganenko \& Stern, 1996) with the solar wind magnetic field GSM components, $B_{y}$ and $B_{z}$ of 7.96 and $-1.84 \mathrm{nT}$, solar wind density of $12.05 \mathrm{~cm}^{-3}$, and solar wind velocity of $368.5 \mathrm{~km} / \mathrm{s}$. As fluctuations in the solar wind were minor in this case, and it was still in the early stage of the growth phase, Tsyganenko produces representative mapping to the inner magnetosphere.

The mapped position of the example Lumikko is just tailward of $X_{\mathrm{GSM}} 8 R_{e}$ moving from $Y_{\mathrm{GSM}} 1.1$ to $2.2 R_{e}$ over $\mathrm{a} \approx 10$-s period as illustrated in Figure 4 . This gives speeds ranging from 1.8 to $4.8 \times 10^{5} \mathrm{~m} / \mathrm{s}$ with an average of $3.4 \times 10^{5} \mathrm{~m} / \mathrm{s}$ (Figure 5). This is comparable to a typical Alfvén speed in the inner plasmasheet $\left(v_{A} \approx 5.5 \times 10^{5} \mathrm{~m} / \mathrm{s}\right)$.

\section{Discussion}

To the best of our knowledge, this is the first report of randomly bidirectional and convection-independent auroral transients in the substorm growth phase. Lumikot associate with quasi-stable growth-phase arcs with a low occurrence rate; probably overlooked in the less extensive growth-phase studies since the work of Lessard et al. (2007). However, the event in this paper was not unique as other examples were also found. Presumably, as faster cadence times have been implemented for auroral imaging, the prevalence of Lumikot becomes more apparent. One observational limitation may be the narrow latitude band.

The sizes of individual Lumikot are in the range of a few kilometers in the north-south and 10-50 km in the east-west direction, that is, meridionally thinner than beads and azimuthally of the order of bead size (Motoba et al., 2012). The $z$ coordinate estimates of $115-120 \mathrm{~km}$ agree with the pulsating aurora heights reported by Partamies et al. (2017), where 557.7-nm median height was $\approx 118 \mathrm{~km}$ before the pulsating started and dropped to $\approx 108 \mathrm{~km}$ during the pulsating aurora. This places the enhancement of Lumikot precipitation energy between those of average recovery-phase aurora and pulsating aurora, embedded in the region of CNA. 
The Lumikot includes enhanced emission embedded in a region of high-energy precipitation, as indicated by CNA of $\approx 0.3 \mathrm{~dB}$ measured by KAIRA. No variation in CNA (to a limit of $\pm 0.05 \mathrm{~dB}$ ) was measured when the Lumikot passed, which may be due to the limited spatial resolution of KAIRA $\left(15^{\circ}\right.$ full width at half maximum at $38.1 \mathrm{MHz}$ ).

As the magnetotail stretches during the growth phase, the auroral arc moves equatorward until the buildup of energy is released at the substorm onset (Lessard et al., 2007). The equatorward arc maps to the inner edge of the plasmasheet where significant gradients in plasma properties may exist (Fukunishi, 1975). However, an inner magnetospheric instability is unlikely to be responsible for bidirectional propagation. Chorus waves have been shown to be responsible for the precipitation during pulsating aurora (Kasahara et al., 2018). While some pulsating patches have been observed to follow the convection (Yang et al., 2017), others, such as the amorphous pulsating aurora (Grono et al., 2017) do not. Lumikot neither follow the convection speed nor the consistency in the direction.

The propagation speed of our sample Lumikot (Example \#1) was found to be $\approx 12 \mathrm{~km} / \mathrm{s}$. This structure was visible in three consecutive images. A majority of other Lumikot were also observed in 3-4 consecutive images, suggesting that our sample speed estimate is representative for the studied cases. The data do not allow the assertion of a preferred direction of motion. So, unlike other previously reported auroral structures, such as streaming auroral pulsating patches (Yamamoto \& Oguti, 1982), azimuthally propagating extensions of north-south aligned arcs (Nishimura et al., 2010), azimuthally moving auroral enhancements (Lyons et al., 2015) or auroral brightening called auroral horn (Koskinen et al., 1990), Lumikot do not show an observable preference in their propagation direction.

The Lumikot phenomenon defies theoretical models which would generate bidirectional propagation of small-scale aurora.

Although the projection of the Lumikot from the ASC images to the $E$ region is quantitatively accurate, the mapping to the plasmasheet should only be seen as qualitatively representative since the Tsyganenko T96 model does not take the dynamics of the magnetosphere and magnetosphere-solar-wind interaction into account. For substorm conditions, this can mean discrepancies between the Tsyganenko model and reality.

According to Nishimura et al. (2016), auroral beads propagate both eastward and westward. Their appearance is fundamentally different from Lumikot as they grow periodically within the growth-phase arc during the minutes prior to the substorm onset and initiate the brightening before the breakup. The relation of Lumikot to the substorm onset instability is much less obvious. However, the beading shows that there are mechanisms producing both propagation directions for small-scale aurora during the growth phase.

Optical pulsating aurora are seen during the growth phase of the substorm both before and after the Lumikot (McKay et al., 2018), suggesting that Lumikot are not "prototype" pulsating aurora. It is not clear whether Lumikot exist in the recovery phase of the substorm, as the complexity of the postbreakup aurora preclude their reliable detection. More detailed relationship between pulsating aurora and Lumikot should be investigated in the future. With the advances in camera sensitivity and sampling rate it has become possible to conduct more comprehensive surveys of transient auroral phenomena. Additionally, new techniques being developed for recovery-phase pulsating aurora (Grono et al., 2017) could be applied to growth-phase research.

\section{Conclusion}

Fast auroral transients, referred to as Lumikot, have been observed during the substorm growth phases. They appear in the region between diffuse aurora and the optically bright growth-phase arc, with pulsating aurora and high-energy particle precipitation leading to CNA. The Lumikot are too small spatially and temporally to greatly modify CNA regardless whether the Lumikot precipitation is energetic enough or not. This region corresponds to CNA and pulsating aurora and, thus, high-energy particle precipitation. Tracing the magnetic field lines maps this band to the border between the outer radiation belt and the plasmasheet.

The Lumikot are several kilometers across and travel east-west parallel to the main growth-phase arc. Both directions of travel have been observed during the same event. They are significant because such bidirectional transients prior to the expansive onset of the auroral breakup challenges the current understanding of substorm instabilities. The magnetospheric dynamics causing the Lumikot are a novel hitherto unknown 
Acknowledgments

We thank the Finnish Meteorological Institute for their operation of the FMI-ASC network. FMI-ASC data are available as keograms at http://www.space.fmi.fi/MIRACLE/ ASC/ and http://gaia-vxo.org. Full resolution images can be requested from Kirsti Kauristie (kirsti.kauristie@fmi.fi). KAIRA was funded by the University of Oulu and FP7 European Regional Development Funds and is operated by Sodankylä Geophysical Observatory and the University of Tromsø, and data are available on request from the PI at http://www.sgo.fi/KAIRA/. Magnetic coordinates and directions were determined using the aacgmv2 Python libraries and the U.S. Department of Commerce, NOAA National Geophysical Data Center online services, https://www.ngdc noaa.gov/geomag-web/. Ancillary data are from the Japanese World Data Centre (http://wdc.kugi.kyoto-u.ac. jp/). The authors thank T. Antonsen and J. Vierinen for useful discussions as well as the suggestions of the anonymous reviewers. D. McKay was supported by the Tromsø Research Foundation. N. Partamies is supported by the Research Council of Norway under CoE Contract 223252 phenomena that can now be used to help understand preonset magnetospheric processes. Lumikot are significant because such bidirectional transients prior to the onset of the auroral breakup may provide important insights to the processes leading to the onset.

\section{References}

Fukunishi, H. (1975). Dynamic relationship between proton and electron auroral substorms. Journal of Geophysical Research, 80, 553-574. https://doi.org/10.1029/JA080i004p00553

Grono, E., Donovan, E., \& Murphy, K. R. (2017). Tracking patchy pulsating aurora through all-sky images. Annales Geophysicae, 35, 777-784. https://doi.org/10.5194/angeo-35-777-2017

Gustavsson, B., Kosch, M., Wong, A., Pedersen, T., Heinselman, C., Mutiso, C., et al. (2008). First estimates of volume distribution of HF-pump enhanced emissions at 6300 and $5577 \AA$ A: A comparison between observations and theory. Annales Geophysicae, 26, 3999-4012 https://doi.org/10.5194/angeo-26-3999-2008

Kasahara, S., Miyoshi, Y., Yokota, S., Mitani, T., Kasahara, Y., Matsuda, S., et al. (2018). Pulsating aurora from electron scattering by chorus waves. Nature, 554, 337-340. https://doi.org/10.1038/nature25505

Koskinen, H. E. J., Pulkkinen, T. I., \& Pellinen, R. J. (1990). Mapping of the auroral horn into the magnetotail. Planetary and Space Science, 38, 1179-1186. https://doi.org/10.1016/0032-0633(90)90025-L

Lessard, M. R., Lotko, W., LaBelle, J., Peria, W., Carlson, C. W., Creutzberg, F., \& Wallis, D. D. (2007). Ground and satellite observations of the evolution of growth phase auroral arcs. Journal of Geophysical Research, 112, A09304. https://doi.org/10.1029/2006JA011794

Lyons, L. R., Nishimura, Y., Gallardo-Lacourt, B., Nicolls, M. J., Chen, S., Hampton, D. L., et al. (2015). Azimuthal flow bursts in the inner plasma sheet and possible connection with saps and plasma sheet earthward flow bursts. Journal of Geophysical Research: Space Physics, 120, 5009-5021. https://doi.org/10.1002/2015JA021023

McKay, D., Partamies, N., \& Vierinen, J. (2018). Pulsating aurora and cosmic noise absorption associated with growth-phase arcs. Annales Geophysicae, 36(1), 59-69. https://doi.org/10.5194/angeo-36-59-2018

McPherron, R. L. (1970). Growth phase of magnetospheric substorms. Journal of Geophysical Research, 75, 5592. https://doi.org/10.1029/ JA075i028p05592

Motoba, T., Hosokawa, K., Kadokura, A., \& Sato, N. (2012). Magnetic conjugacy of northern and southern auroral beads. Geophysical Research Letters, 39, L08108. https://doi.org/10.1029/2012GL051599

Nishimura, Y., Lyons, L., Zou, S., Angelopoulos, V., \& Mende, S. (2010). Substorm triggering by new plasma intrusion: THEMIS all-sky imager observations. Journal of Geophysical Research, 115, A07222. https://doi.org/10.1029/2009JA015166

Nishimura, Y., Yang, J., Pritchett, P. L., Coroniti, F. V., Donovan, E. F., Lyons, L. R., et al. (2016). Statistical properties of substorm auroral onset beads/rays. Journal of Geophysical Research: Space Physics, 121, 8661-8676. https://doi.org/10.1002/2016JA022801

Ono, T., Hirasawa, T., \& Meng, C. I. (1987). Proton auroras observed at the equatorward edge of the duskside auroral oval. Geophysical Research Letters, 14, 660-663. https://doi.org/10.1029/GL014i006p00660

Partamies, N., Whiter, D., Kadokura, A., Kauristie, K., Nesse Tyssøy, H., Massetti, S., et al. (2017). Occurrence and average behavior of pulsating aurora. Journal of Geophysical Research: Space Physics, 122, 5606-5618. https://doi.org/10.1002/2017JA024039

Sangalli, L., Partamies, N., Syrjäsuo, M., Enell, C.-F., Kauristie, K., \& Mläkinen, S. (2011). Performance study of the new EMCCD-based all-sky cameras for auroral imaging. International Journal of Remote Sensing, 32, 2987-3003. https://doi.org/10.1002/2017JA024039

Shepherd, S. G. (2014). Altitude-adjusted corrected geomagnetic coordinates: Definition and functional approximations. Journal of Geophysical Research: Space Physics, 119, 7501-7521. https://doi.org/10.1002/2014JA020264

Tsyganenko, N. A., \& Stern, D. P. (1996). Modeling the global magnetic field of the large-scale Birkeland current systems. Journal of Geophysical Research, 101, 27,187-27,198. https://doi.org/10.1029/96JA02735

Wing, S., Gkioulidou, M., Johnson, J. R., Newell, P. T., \& Wang, C.-P. (2013). Auroral particle precipitation characterized by the substorm cycle. Journal of Geophysical Research: Space Physics, 118, 1022-1039. https://doi.org/10.1002/jgra.50160

Yamamoto, T., \& Oguti, T. (1982). Recurrent fast motions of pulsating auroral patches. I-A case study on optical and quantitative characteristics during a slightly active period. Journal of Geophysical Research, 87, 7603-7614. https://doi.org/10.1029/JA087iA09p07603

Yang, B., Donovan, E., Liang, J., \& Spanswick, E. (2017). A statistical study of the motion of pulsating aurora patches: Using the THEMIS all-sky imager. Annales Geophysicae, 35, 217-225. https://doi.org/10.5194/angeo-35-217-2017 